STRUCTURAL BIOLOGY COMMUNICATIONS

ISSN 2053-230X

\section{Crystal structure of human CRM1, covalently modified by 2-mercaptoethanol on Cys528, in complex with RanGTP}

\author{
Alaa Shaikhqasem, ${ }^{\text {a }}$ Kerstin Schmitt, ${ }^{\text {b }}$ Oliver Valerius ${ }^{\mathrm{b}}$ and Ralf Ficner $^{\mathrm{a} *}$ \\ a'Department for Molecular Structural Biology, Georg-August-Universität Göttingen, Justus-von-Liebig Weg 11, \\ 37077 Göttingen, Germany, and ${ }^{\mathbf{b}}$ Department of Molecular Microbiology and Genetics, Georg-August-Universität \\ Göttingen, Grisebachstrasse 8, 37077 Göttingen, Germany. *Correspondence e-mail: rficner@gwdg.de
}

Received 5 January 2021

Accepted 21 February 2021

Edited by N. Sträter, University of Leipzig, Germany

Keywords: nuclear export; cancer; exportin 1; cysteine modification.

PDB reference: human CRM1 covalently modified by 2-mercaptoethanol, 7 b5 1

Supporting information: this article has supporting information at journals.iucr.org/f

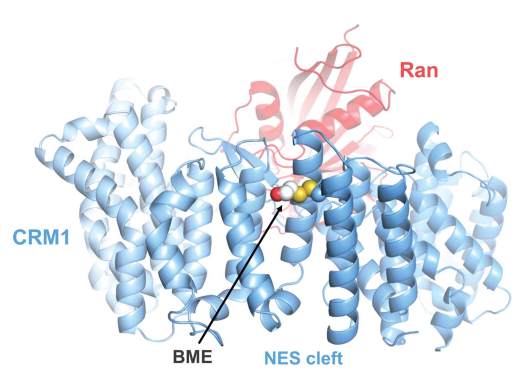
OPEN ○ ACCESS
CRM1 is a nuclear export receptor that has been intensively targeted over the last decade for the development of antitumor and antiviral drugs. Structural analysis of several inhibitor compounds bound to CRM1 revealed that their mechanism of action relies on the covalent modification of a critical cysteine residue (Cys528 in the human receptor) located in the nuclear export signalbinding cleft. This study presents the crystal structure of human CRM1, covalently modified by 2-mercaptoethanol on Cys528, in complex with RanGTP at $2.58 \AA$ resolution. The results demonstrate that buffer components can interfere with the characterization of cysteine-dependent inhibitor compounds.

\section{Introduction}

CRM1 (chromosomal region maintenance 1) is an essential nucleocytoplasmic transport receptor that mediates the nuclear export of a wide range of proteins and ribonucleoprotein complexes (Güttler \& Görlich, 2011; Kırlı et al., 2015). CRM1-mediated transport is dependent on the cooperative binding of the small GTPase Ran in its GTP-bound form (RanGTP) and of the cargo protein to the export receptor, forming a trimeric complex that traverses the nuclear pore complex. The overall architecture of CRM1 consists of a ringlike structure that is composed of 21 HEAT repeats, each consisting of two antiparallel $\alpha$-helices, A and B, connected via a short linker loop (Monecke et al., 2009; Dong et al., 2009). CRM1 recognizes cargo proteins by their leucine-rich nuclear export signal (NES) peptide. The NES peptide contains four or five hydrophobic residues that bind specifically into five hydrophobic pockets ( $\Phi 0-\Phi 4)$ located in the cleft between HEAT repeats $11 \mathrm{~A}$ and $12 \mathrm{~A}$ (referred to as the NES-binding cleft; la Cour et al., 2004; Fung et al., 2017). Overexpression of CRM1 has been observed in several cancers, and it has been identified as the major nuclear exporter for several oncoproteins, growth regulators and suppressor proteins such as p53, p21, BRCA1/2, Rb and FOXO, which lead to the initiation and progression of cancer (Hill et al., 2014; Faustino et al., 2007; Turner et al., 2012; Watt \& Leaner, 2010). Furthermore, CRM1 plays a key role in several viral diseases as it is co-opted by viruses such as influenza, rabies virus $\mathrm{P}$ and HIV for the nuclear export of their RNA and ribonucleoprotein (RNP) complexes. This renders CRM1 an interesting drug target for therapeutic intervention in several cancers and viral diseases (Mathew \& Ghildyal, 2017; Dickmanns et al., 2015).

Leptomycin B (LMB), a natural compound that consists of a polyketide chain with an $\alpha, \beta$-unsaturated lactone ring, was the first CRM1 inhibitor to be discovered (Hamamoto et al., 
1985; Kudo et al., 1998). Clinical tests revealed severe side effects and high toxicity (Newlands et al., 1996), which induced a continuous search for and the development of alternative natural and synthetic compounds that could be used in CRM1 inhibition (Tamura et al., 2010; Kau et al., 2003; Liu et al., 2015; Daelemans et al., 2002; Bonazzi et al., 2010; Sakakibara et al., 2011; Mathew \& Ghildyal, 2017). Structural analysis by means of X-ray crystallography defined the molecular basis of CRM1 inhibition. Furthermore, it has been used as a reliable approach for the development of novel CRM1 inhibitors (Kalid et al., 2012; Sun et al., 2013; Tian et al., 2020). The crystallographic analysis of several natural and synthetic compounds bound to CRM1 defined a general mechanism of inhibition by the covalent modification of a reactive cysteine residue located in the NES-binding cleft of human CRM1 (Cys528). The binding of inhibitor compounds, mediated by the reactive cysteine, interferes with the binding of the NES peptide and prevents the formation of a stable export complex. As the human protein failed to crystallize in complex with inhibitors, CMR1 from the yeast Saccharomyces cerevisiae $\left({ }^{S c} \mathrm{CRM} 1\right)$ was genetically modified to incorporate the reactive cysteine (T539C) and was used to crystallize CRM1 in complex with several inhibitor compounds (Lapalombella et al., 2012; Etchin et al., 2013; Haines et al., 2015; Hing et al., 2016; Tian et al., 2020). Recently, we developed a crystallization approach using a stabilized variant of human CRM1 $\left({ }^{H s} \mathrm{CRM} 1\right)$, with which we succeeded in solving the crystal structure of LMB bound to ${ }^{H s} \mathrm{CRM} 1$ in complex with RanGTP (Shaikhqasem et al., 2020). We furthermore investigated the molecular mechanism of several novel CRM1 inhibitors, including the compounds C3 [IUPAC name 2-[[1,1,1,3,3,3hexafluoro-2-[(2-fluorobenzoyl)amino]propan-2-yl]amino]-4,5, 6,7-tetrahydro-1-benzothiophene-3-carboxamide\}, C6 \{IUPAC name 4-(4-chlorophenyl)sulfonyl- $N, N$-dimethyl-2-(3,4,5-trimethoxyphenyl)-1,3-oxazol-5-amine\} and C10 \{IUPAC name 5-methoxythieno[3,2-d][1,2]thiazole\} (Fetz et al., 2009; Shaikhqasem et al., 2020). This investigation revealed that these three compounds disrupt CRM1-NES interaction due to their binding to ${ }^{H s} \mathrm{CRM} 1$ at different rates in a Cys528dependent manner (Shaikhqasem et al., 2020). As the compound $\mathrm{C} 6$ exhibited the highest binding affinity among the tested compounds, we tried to crystallize it in complex with ${ }^{H s}$ CRM1 following the same approach as used for LMB. However, crystallographic analysis revealed an unexpected modification of the reactive cysteine in several data sets collected from different crystals of potential ${ }^{H s}$ CRM1-C6 complexes. Here, we present structural insight into the covalent modification of Cys528 of ${ }^{H s} \mathrm{CRM} 1$ by 2-mercaptoethanol (BME), which was introduced as a buffer component during protein purification.

\section{Materials and methods}

\subsection{Macromolecule production}

A HEAT9 loop mutant $\left({ }^{430} \mathrm{VLV}^{432}\right.$ to AAA) of C-terminally truncated ( $\alpha$-helix; $\Delta 1037-1071){ }^{H s} \mathrm{CRM} 1\left({ }^{H s} \mathrm{CRM} 1{ }^{\Delta}\right)$ was expressed and purified as described previously (Shaikhqasem et al., 2020). The purification buffer in the last purification step contained $6 \mathrm{~m} M$ BME. Human RanGTP ${ }^{1-180, Q 69 \mathrm{~L}}$ (Monecke et al., 2009) was prepared as described in Port et al. (2015). Macromolecule-production information for both CRM1 and Ran is summarized in Table 1.

\subsection{Crystallization}

For crystallization, the complex was prepared following the same protocol as used to prepare the ${ }^{H s} \mathrm{CRM} 1^{\Delta}$-RanGTPLMB complex (Shaikhqasem et al., 2020) with the exception of the addition of C6 (synthesized by ChemBridge Corporation, USA) in a ten-molar excess to CRM1 to counter its lower binding affinity in comparison with LMB. Single crystals with moderate diffraction quality grew within 3-6 days in the commercial crystallization buffer Morpheus H10 (Gorrec, 2009; Table 2) when mixed in a 1:1 ratio with the complex concentrated to $3 \mathrm{mg} \mathrm{ml}^{-1}$. Crystallization information is summarized in Table 2.

\subsection{Data collection and processing}

X-ray diffraction data were collected on EMBL beamline P13 at PETRA III, DESY, Hamburg, Germany equipped with a PILATUS 6M detector. The collected data were indexed, processed and scaled using the XDS package (Kabsch, 2010). The data set revealed an orthorhombic lattice, with unit-cell parameters $a=121.11, b=150.59, c=231.97 \AA$, belonging to space group 1222 (Table 3 ).

\subsection{Structure solution and refinement}

The structure was solved by molecular replacement with Phaser (McCoy et al., 2007) using the crystal structure of the ${ }^{H s} \mathrm{CRM} 1{ }^{\Delta}$-RanGTP-LMB complex (PDB entry 6tvo; Shaikhqasem et al., 2020) as the search model. The structure was refined to reasonable $R$ factors (Table 4) by iterative cycles of refinement and manual rebuilding in REFMAC5 (Murshudov et al., 2011) and Coot (Emsley et al., 2010), respectively. Water molecules were added with consideration of hydrogen-bond restraints and only if both $m F_{\mathrm{o}}-D F_{\mathrm{c}}$ and $2 m F_{\mathrm{o}}-D F_{\mathrm{c}}$ electron-density map peaks were simultaneously present at levels of $3.0 \sigma$ and greater than $1.0 \sigma$, respectively. BME was modeled manually in Coot. A polder OMIT map (Liebschner et al., 2017), generated by omitting both BME and Cys528, verified the presence of the Cys-BME conjugate. The root-mean-square deviation (r.m.s.d.) measurements were calculated using LSQMAN (Kleywegt, 1999). Figures were generated with PyMOL (version 1.8; Schrödinger).

\subsection{Liquid chromatography-mass spectrometry analysis}

Four samples were prepared for liquid chromatographymass spectrometry (LC-MS): ${ }^{H s} \mathrm{CRM} 1{ }^{\Delta},{ }^{H s} \mathrm{CRM} 1{ }^{\Delta}$ mixed with crystallization buffer (Morpheus H10) and incubated overnight at $4{ }^{\circ} \mathrm{C}$ to mimic the crystallization environment, crystals grown using the ${ }^{H s} \mathrm{CRM} 1{ }^{\Delta}$-RanGTP-C6 complex and crystals used for diffraction experiments. Cysteine-reactive chemicals, reducing agents and cysteine-modifying steps were 
Table 1

Macromolecule-production information.

\begin{tabular}{|c|c|c|}
\hline Macromolecule & ${ }^{H s} \mathrm{CRM}{ }^{\Delta}$ & ${ }^{H s} \operatorname{Ran}^{1-180, \mathrm{Q} 69 \mathrm{~L}}$ \\
\hline $\begin{array}{l}\text { Source organism } \\
\text { Expression vector } \\
\text { Expression host } \\
\text { Complete amino-acid sequence } \\
\text { of the construct produced }\end{array}$ & $\begin{array}{l}\text { Homo sapiens } \\
\text { pET-21a } \\
\text { E. coli BL21(DE3) } \\
\text { MASMTGGQQMGRGSMPAIMTMLADHAARQLLDFSQKLDINLLD } \\
\text { NVVNCLYHGEGAQQRMAQEVLTHLKEHPDAWTRVDTILEFS } \\
\text { QNMNTKYYGLQILENVIKTRWKILPRNQCEGIKKYVVGLII } \\
\text { KTSSDPTCVEKEKVYIGKLNMILVQILKQEWPKHWPTFISD } \\
\text { IVGASRTSESLCQNNMVILKLLSEEVFDFSSGQITQVKSKH } \\
\text { LKDSMCNEFSQIFQLCQFVMENSQNAPLVHATLETLLRFLN } \\
\text { WIPLGYIFETKLISTLIYKFLNVPMFRNVSLKCLTEIAGVS } \\
\text { VSQYEEQFVTLFTLTMMQLKQMLPLNTNIRLAYSNGKDDEQ } \\
\text { NFIQNLSLFLCTFLKEHDQLIEKRLNLRETLMEALHYMLLV } \\
\text { SEVEETEIFKICLEYWNHLAAELYRESPFSTSASPLLSGSQ } \\
\text { HFDVPPRRQLYLPMLFKVRLLMVSRMAKPEEAAAVENDQGE } \\
\text { VVREFMKDTDSINLYKNMRETLVYLTHLDYVDTERIMTEKL } \\
\text { HNQVNGTEWSWKNLNTLCWAIGSISGAMHEEDEKRFLVTVI } \\
\text { KDLLGLCEQKRGKDNKAIIASNIMYIVGQYPRFLRAHWKFL } \\
\text { KTVVNKLFEFMHETHDGVQDMACDTFIKIAQKCRRHFVQVQ } \\
\text { VGEVMPFIDEILNNINTIICDLQPQQVHTFYEAVGYMIGAQ } \\
\text { TDQTVQEHLIEKYMLLPNQVWDSIIQQATKNVDILKDPETV } \\
\text { KQLGSILKTNVRACKAVGHPFVIQLGRIYLDMLNVYKCLSE } \\
\text { NISAAIQANGEMVTKQPLIRSMRTVKRETLKLISGWVSRSN } \\
\text { DPQMVAENFVPPLLDAVLIDYQRNVPAAREPEVLSTMAIIV } \\
\text { NKLGGHITAEIPQIFDAVFECTLNMINKDFEEYPEHRTNEF } \\
\text { LLLQAVNSHCFPAFLAIPPTQFKLVLDSIIWAFKHTMRNVA } \\
\text { DTGLQILFTLLQNVAQEEAAAQSYQTYFCDILQHIFSVVT } \\
\text { DTSHTAGLTMHASILAYMFNLVEEGKISTSLNPGNPVNNQI } \\
\text { FLQEYVANLLKSAFPHLQDAQVKLFVTGLFSLNQDIPAFKE } \\
\text { HLRDFLVQIKEFAGEDTSDLFLERSRSHHHHHH }\end{array}$ & $\begin{array}{l}\text { Homo sapiens } \\
\text { pQE80 } \\
\text { E. coli BL21(DE3)pLysS } \\
\text { MGMAAQGEPQVQFKLVLVGDGGTGKTTFVKRHLTGEFEKKYVA } \\
\text { TLGVEVHPLVFHTNRGPIKFNVWDTAGLEKFGGLRDGYYIQ } \\
\text { AQCAI IMFDVTSRVTYKNVPNWHRDLVRVCENIPIVLCGNK } \\
\text { VDIKDRKVKAKS IVFHRKKNLQYYDISAKSNYNFEKPFLWL } \\
\text { ARKLIGDPNLEFVAMP }\end{array}$ \\
\hline
\end{tabular}

Table 2

Crystallization.

\begin{tabular}{ll}
\hline Method & Vapor diffusion \\
Plate type & Sitting drop \\
Temperature $(\mathrm{K})$ & 277.15 \\
Protein concentration $\left(\mathrm{mg} \mathrm{ml}^{-1}\right)$ & 3 \\
Buffer composition of protein & $50 \mathrm{~m} M$ HEPES pH 7.8, 130 m $M \mathrm{NaCl}$, \\
$\quad$ solution & $2 \mathrm{~m} M \mathrm{MgCl}_{2}, 6 \mathrm{~m} M$ 2-mercaptoethanol \\
Composition of reservoir & $10 \%(w / v)$ PEG 8000, 20\% $(v / v)$ ethylene \\
$\quad$ solution & glycol, $100 \mathrm{~m} M$ Bicine-Tris base pH 8.5, \\
& $20 \mathrm{~m} M$ sodium DL-glutamate, $20 \mathrm{~m} M$ \\
& DL-alanine, 20 m $M$ glycine, 20 m $M$ \\
Volume and ratio of drop & DL-lysine-HCl, $20 \mathrm{~m} M$ DL-serine \\
Volume of reservoir $(\mu \mathrm{l})$ & $0.5 \mu l ; 1: 1$ ratio \\
\hline
\end{tabular}

avoided throughout the entire procedure. Equal volumes of the protein solutions were mixed with $2 \times$ SDS sample buffer (62.5 $\mathrm{m} M$ Tris- $\mathrm{HCl} \mathrm{pH}$ 6.8, 2.5\% SDS, $0.002 \%$ bromophenol blue, $10 \%$ glycerol). Crystals not exposed to X-ray radiation were transferred and dissolved in a drop containing $1 \times$ SDS sample buffer, while crystals used in diffraction experiments were carefully thawed in a drop of water and were then mixed with an equal volume of $2 \times$ SDS sample buffer. Afterwards, samples were boiled at $95^{\circ} \mathrm{C}$ for $5 \mathrm{~min}$ and applied onto an SDS-PAGE gel for brief separation. Protein-containing bands were cut and subjected to trypsin (SERVA Electrophoresis, catalogue No. 37283.01) digestion according to Shevchenko et al. (1996). Desalting of tryptic peptides prior to LC-MS was performed via StageTips according to the protocol described by Rappsilber et al. (2007). $2 \mu \mathrm{l}$ of each sample was subjected to reverse-phase liquid chromatography for peptide separa-
Table 3

Data collection and processing.

Values in parentheses are for the outer shell.

Diffraction source

Wavelength $(\AA)$

Temperature (K)

Detector

Crystal-to-detector distance ( $\mathrm{mm}$ )

Rotation range per image $\left(^{\circ}\right)$

Total rotation range $\left({ }^{\circ}\right)$

Exposure time per image (s)

Space group

$a, b, c(\AA)$

$\alpha, \beta, \gamma\left({ }^{\circ}\right)$

Resolution range $(\AA)$

Total No. of reflections

No. of unique reflections

Completeness (\%)

Multiplicity

$\langle I / \sigma(I)\rangle$

$R_{\text {meas }}$

$\mathrm{CC}_{1 / 2}(\%)$

Overall $B$ factor from Wilson plot $\left(\AA^{2}\right)$

P13, PETRA III, DESY 0.9762

100

PILATUS 6M

576.1

0.1

222

0.05

I222

$121.11,150.59,231.97$

90, 90, 90

$126.31-2.58(2.74-2.58)$

$519292(48302)$

65209 (9016)

$97.5(84.3)$

$7.96(5.36)$

$16.60(1.95)$

$0.083(0.712)$

$99.9(81.7)$

64.7

tion using an RSLCnano Ultimate 3000 system (Thermo Fisher Scientific). The peptides were loaded onto an Acclaim PepMap 100 pre-column $(100 \mu \mathrm{m} \times 2 \mathrm{~cm}, \mathrm{C} 18,5 \mu \mathrm{m}, 100 \AA$ A Thermo Fisher Scientific) with $0.07 \%$ trifluoroacetic acid at a flow rate of $20 \mu \mathrm{l} \mathrm{min}^{-1}$ for $3 \mathrm{~min}$. Analytical separation of the peptides was performed on an Acclaim PepMap RSLC column $(75 \mu \mathrm{m} \times 50 \mathrm{~cm}, \mathrm{C} 18,2 \mu \mathrm{m}, 100 \AA$; Thermo Fisher

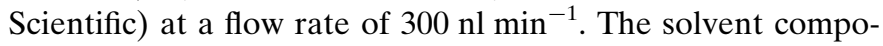
sition was gradually changed over 94 min from $96 \%$ solvent $A$ 
Table 4

Structure solution and refinement.

Values in parentheses are for the outer shell.

\begin{tabular}{ll}
\hline Resolution range $(\AA)$ & $126.31-2.58(2.65-2.58)$ \\
Completeness $(\%)$ & 97.5 \\
$\sigma$ Cutoff & $F>0.0 \sigma(F)$ \\
No. of reflections, working set & $61972(3242)$ \\
No. of reflections, test set & $3237(149)$ \\
Final $R_{\text {cryst }}$ & $0.214(0.544)$ \\
Final $R_{\text {free }}$ & $0.250(0.583)$ \\
No. of non-H atoms & \\
$\quad$ Protein & 9613 \\
$\quad$ Ligand & 37 \\
$\quad$ Water & 136 \\
$\quad$ Total & 9786 \\
R.m.s. deviations & \\
$\quad$ Bonds $(\AA)$ & 0.008 \\
$\quad$ Angles $\left({ }^{\circ}\right)$ & 1.605 \\
Average $B$ factors $\left(\AA^{2}\right)$ & \\
$\quad$ Protein & 62.2 \\
$\quad$ Ligand & 50.2 \\
$\quad$ Water & 59.9 \\
Ramachandran plot & \\
$\quad$ Most favored $(\%)$ & 97.0 \\
$\quad$ Allowed $(\%)$ & 3.0 \\
PDB code & $7 \mathrm{~b} 51$ \\
\hline
\end{tabular}

( $0.1 \%$ formic acid) and $4 \%$ solvent $B(80 \%$ acetonitrile, $0.1 \%$ formic acid) to $10 \%$ solvent $B$ within 2 min, to $30 \%$ solvent $B$ within the next $58 \mathrm{~min}$, to $45 \%$ solvent $B$ within the following $22 \mathrm{~min}$ and to $90 \%$ solvent $B$ within the last $12 \mathrm{~min}$ of the gradient. All solvents and acids were of Optima grade for LCMS (Thermo Fisher Scientific). Eluting peptides were ionized online by nano-electrospray using a Nanospray Flex Ion Source (Thermo Scientific) at $1.5 \mathrm{kV}$ (liquid junction) and transferred into a Q Exactive HF mass spectrometer (Thermo Fisher Scientific). Full scans in the mass range $300-1650 \mathrm{~m} / \mathrm{z}$ were recorded at a resolution of 30000 followed by datadependent top $10 \mathrm{HCD}$ fragmentation at a resolution of 15000 (dynamic exclusion enabled). LC-MS method programming and data acquisition were performed with the XCalibur 4.0 software (Thermo Fisher Scientific).

MS/MS2 data were searched against an Escherichia coli specific protein database (UniProt Proteome ID UP000000625) that additionally contained the CRM1 sequence using MaxQuant 1.6.0.16 (Cox \& Mann, 2008). The digestion mode was trypsin/P, and the maximum number of missed cleavage sites was set to two. Oxidation at methionine and $\mathrm{N}$-terminal protein acetylation were set as variable modifications. A search for dependent peptides was performed to identify additional peptide modifications. The mass tolerances of precursors and fragment ions were 4.5 p.p.m. and 20 p.p.m. (HCD), respectively. False-discovery rates were calculated using the revert decoy mode, and the threshold for peptide-sequence matches as well as protein identifications was 0.01. MaxQuant output data were further evaluated using Perseus 1.6.0.7 (Tyanova et al., 2016). The dependent-peptide search provided evidence for the presence of a DeStreak (2-mercaptoethanol; BME) modification ( $\Delta$ mass of 75.9983$)$ at the cysteine residue of the CRM1 peptide DLLGLCEQK (Asp523-Lys531). Based on this result, the data were searched against the same database as before using Proteome Discoverer 2.2.0.388 with the SequestHT search algorithm and the DeStreak modification at cysteines as a variable modification. Precursor mass tolerance and fragment mass tolerances were 10 p.p.m. and $0.02 \mathrm{Da}$, respectively. The digestion mode and false-discovery rate were the same as for the MaxQuant analysis.

\section{Results \\ 3.1. Identification of the modification of Cys528 by 2-mercaptoethanol}

The main aim of the performed crystallization experiment was to gain structural insight into the interaction of C6 with CRM1. Our recent results showed that the compound exhibits a reduced inhibitory activity when Cys528 is changed to a serine, suggesting that the compound is binding to or is in the vicinity of the reactive cysteine in the NES-binding cleft (Shaikhqasem et al., 2020). Structure refinement of several data sets obtained from crystals grown using the ${ }^{H s} \mathrm{CRM} 1{ }^{\Delta}{ }^{H s}$ RanGTP-C6 complex displayed excess difference electron density at Cys528 (Fig. 1a). Nevertheless, the positive density in the $m F_{\mathrm{o}}-D F_{\mathrm{c}}$ map ( $3 \sigma$ level) was much smaller in size than expected for the compound $\mathrm{C} 6$ and could not be explained by the compound. The center of the

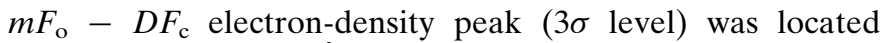
within a distance of $2 \AA$ from the $\mathrm{S}$ atom of Cys528, indicating a possible covalent modification. In order to confirm the type of modification and to elucidate whether it was introduced prior to crystallization or by synchrotron radiation, LC-MS analysis was performed for samples prepared from purified ${ }^{H s} \mathrm{CRM} 1{ }^{\Delta}$, the ${ }^{H s} \mathrm{CRM} 1{ }^{\Delta}$-RanGTP-C6 complex mixed with crystallization buffer (Morpheus H10), complex crystals that were not used for diffraction experiments and crystals exposed to synchrotron radiation.

The results of the LC-MS analysis revealed a mass difference of $75.9983 \mathrm{Da}$ at the cysteine residue within the tryptic peptide DLLGLCEQK (the reactive cysteine Cys528; Fig. 2). The observed shift corresponds to a Cys-BME conjugate, revealing the covalent modification of Cys528 by BME, known as a DeStreak modification (Kim et al., 2015). Although BME was introduced into the CRM1 buffer as a reducing agent during protein purification, the complex mixed with the crystallization buffer and the crystals of the complex before and after exposure to synchrotron radiation were more BMEbound compared with the purified protein. This further confirms that the reactivity of BME towards the cysteine is induced by the crystallization buffer conditions and that the observed excess electron-density map is not related to radiation damage caused by synchrotron radiation.

Crystallographic refinement of the atomic model containing the Cys528-BME conjugate explained the excess electron density (Figs. $1 b$ and $1 c$ ), which was also further supported by a polder OMIT map calculated with Phenix (Liebschner et al., 2019; Fig. 1d). While the disulfide bond fits the electrondensity map, the freely rotatable methyl hydroxy moiety was 
not visible in the density, indicating that it is most likely to be disordered.

\subsection{Crystal structure of ${ }^{\mathrm{Hs}} \mathrm{CRM} 1$ covalently modified by BME} in the NES-binding cleft

The structure presented here was obtained using the previously established protocol employed for crystallization of the ${ }^{H s}$ CRM1 ${ }^{\Delta}$-RanGTP-LMB complex. Similar to the structure of the LMB complex, the CRM1 molecule demonstrates an overall toroid-like compact conformation that results from the typical HEAT-repeat tandem arrangement (Fig. 3a). Ran is bound in the inner core of the CRM1 toroid and its binding is stabilized by the acidic loop, which is arranged in a seatbeltlike structure. The NES-binding cleft, with BME covalently bound to Cys528, is in an open conformation when compared with the unliganded cleft of ${ }^{S c} \mathrm{CRM} 1$ in complex with Ran and RanBP1 ( $C^{\alpha}$ r.m.s.d. of $1.545 \AA$ for the cleft residues 509-576 and the corresponding residues of ${ }^{S c}$ CRM1; Fig. $3 b$ ). However, the BME-bound cleft exhibits a slightly narrower conformation when compared with the LMB-bound cleft of the same CRM1 variant ( $\mathrm{C}^{\alpha}$ r.m.s.d. of $0.897 \AA$ for cleft residues 509576; Fig. 3c) and a further narrowed conformation when compared with the CRM1 cleft occupied by an NES peptide ( $C^{\alpha}$ r.m.s.d. of $0.983 \AA$ for cleft residues 509-576; Fig. $3 d$ ). The observed differences in the conformation of the NES-binding cleft indicate that its conformational plasticity allows it to accommodate ligands of different sizes and variable structures. Nevertheless, as presented here, the covalent binding of BME to Cys528 hinders the binding of the ligand of interest, which indicates that BME should be excluded from both the proteinpreparation and crystallization processes. Covalent modification of cysteine by BME has been reported in several protein structures [for example, PDB entries 5xhe (Mathur et al., 2018), 2jpt (Zhukov et al., 2008) and 1nzu (Nicola et al., 2005)] and it has been shown to interfere significantly with ligand

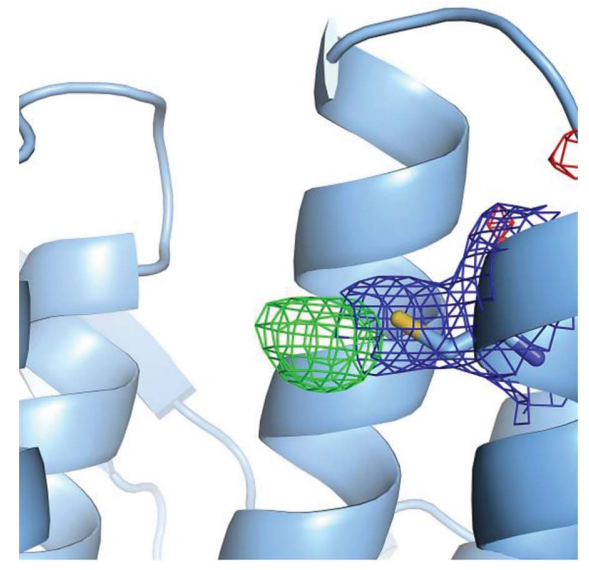

$(a)$

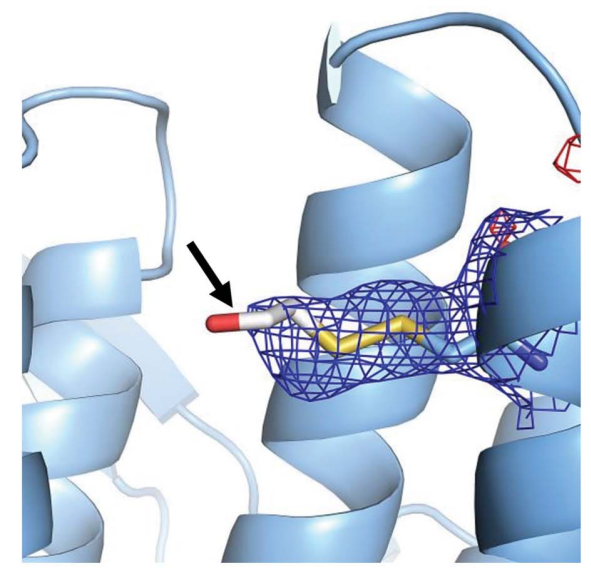

(c)

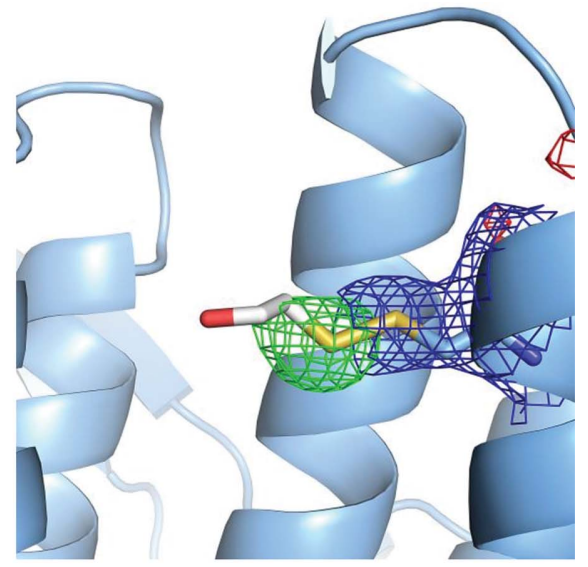

$(b)$

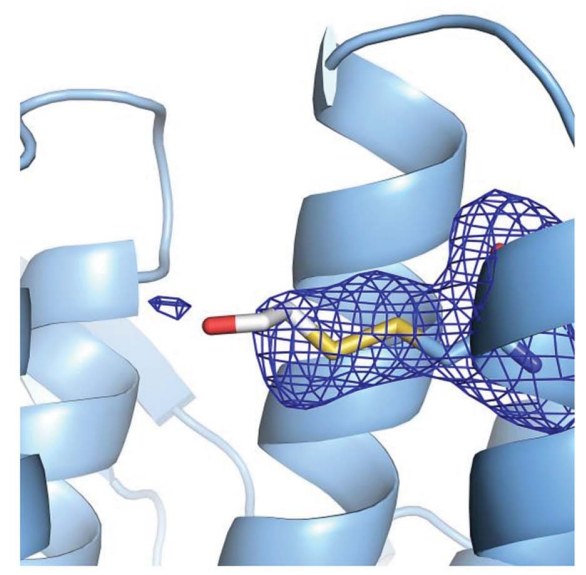

(d)

Figure 1

The modification of Cys528 by 2-mercaptoethanol (BME). $2 m F_{\mathrm{o}}-D F_{\mathrm{c}}$ (contoured at $1.0 \sigma$ in blue) and $m F_{\mathrm{o}}-D F_{\mathrm{c}}$ (contoured at $3.0 \sigma$ in green and $-3.0 \sigma$ in red) difference electron-density maps around Cys528 generated after model refinement with unmodified cysteine in $(a)$ and $(b)$ and after refinement of the covalently modified Cys528-BME conjugate model in $(c)$. Nonmodified cysteine demonstrates an excess electron-density map representing a pronounced positive peak in the $m F_{\mathrm{o}}-D F_{\mathrm{c}}$ map $(a)$. The $\mathrm{S}$ atom of the modeled BME occupies the center of the excess electron-density map peak observed close to Cys528 (b). (c) Crystallographic refinement of the atomic model containing the Cys528-MBE conjugate. Neither positive nor negative peaks in the $m F_{\mathrm{o}}-D F_{\mathrm{c}}$ map could be observed at contour levels of $3.0 \sigma$ and $-3.0 \sigma$, respectively. The methyl hydroxy moiety (indicated by an arrow) was not visible in the electron-density map due to its flexibility (rotational freedom). (d) A polder OMIT map of the Cys528-BME conjugate (contoured at $2.2 \sigma$ in blue) confirms that Cys528 modification by BME explains the excess electron-density peak. 
binding (Mathur et al., 2018) or to strongly influence protein functionality (Nicola et al., 2005; Zhukov et al., 2008).

\section{Discussion}

Recently, we conceived a crystallization approach that allowed us to gain structural insight into the cysteine-mediated covalent inhibition of human CRM1 by the classical nuclear export inhibitor LMB (Shaikhqasem et al., 2020). However, when the same method was applied to crystallize a novel inhibitor compound known as C6 (Fetz et al., 2009), the obtained crystal structure revealed the unexpected covalent modification of Cys 528 by BME (Figs. 1 and 2), which became an obstacle in obtaining structural information on C6-CRM1 interactions. Although BME was used as a reducing agent during CRM1 purification, the results of mass-spectrometric analysis of the purified protein before and after mixing with crystallization buffer indicate that the modification is induced by the crystallization buffer conditions. The reactivity of both the cysteine and BME is most probably induced due to the higher $\mathrm{pH}$ of the crystallization buffer (Poole, 2015). The utilized crystallization condition (Morpheus condition $\mathrm{H} 10$ ) has a $\mathrm{pH}$ of 8.5 , which is maintained by a mixture of Bicine and Tris added to $100 \mathrm{~m} M$, while the purification buffer has a $\mathrm{pH}$ of 7.8 maintained by $50 \mathrm{~m} M$ HEPES (Table 2). A pH change can induce reactivity of the cysteine by the deprotonation of its thiol moiety $(R \mathrm{SH})$ when increased above its $\mathrm{p} K_{\mathrm{a}}$. The cysteine side chain has a default $\mathrm{p} K_{\mathrm{a}}$ value of 9 . However, the $\mathrm{p} K_{\mathrm{a}}$ value can be significantly affected by the microenvironment of the cysteine (Bhatnagar \& Bandyopadhyay, 2018; Klomsiri et al., 2011). For example, metal-binding enzymatic cysteines were shown to exhibit a lower range of $\mathrm{p} K_{\mathrm{a}}$ values $(8.1 \pm 2.2)$ when buried in a hydrophobic cluster (Bhatnagar \& Bandyopadhyay, 2018). Furthermore, BME exhibits decreased stability as the $\mathrm{pH}$ increases, which can lead to the formation of covalent adducts with surface cysteines (Wingfield, 1995). Nevertheless, the covalent adduct of Cys528 and BME was only observed when C6 was used for crystallization and not when LMB was used; the latter was clearly defined in the electron-density map of the crystal structure of the ${ }^{H s} \mathrm{CRM}^{\Delta}{ }^{\Delta}$-RanGTP-LMB complex (Shaikhqasem et al., 2020). This can be explained by the tight irreversible covalent binding of LMB to Cys528. Surprisingly, crystal structures of LMB bound to human and yeast CRM1 export receptors have revealed that the lactone ring of LMB is hydrolyzed upon binding (Shaikhqasem et al., 2020; Sun et al., 2013). Polar interactions with surrounding positively charged residues have been shown to stabilize the lactone ring in an open conformation, which renders the covalent conjunction with LMB irreversible (Sun et al., 2013). The incubation of CRM1 with an

\begin{tabular}{ccccccc}
\hline Order & $\begin{array}{c}\mathrm{b}^{+} \\
(\mathrm{m} / \mathrm{z})\end{array}$ & $\begin{array}{c}\mathrm{b}^{2+} \\
(\mathrm{m} / \mathrm{z})\end{array}$ & Sequence & $\begin{array}{c}\mathrm{y}^{+} \\
(\mathrm{m} / \mathrm{z})\end{array}$ & $\begin{array}{c}\mathrm{y}^{2+} \\
(\mathrm{m} / \mathrm{z})\end{array}$ & Order \\
\hline 1 & 116.03422 & 58.52075 & $\mathrm{D}$ & & & 9 \\
2 & 229.11828 & 115.06278 & $\mathrm{~L}$ & 979.49510 & 490.25119 & 8 \\
3 & 342.20235 & 171.60481 & $\mathrm{~L}$ & 866.41104 & 433.70916 & 7 \\
4 & 399.22381 & 200.11554 & $\mathrm{G}$ & 753.32697 & 377.16712 & 6 \\
5 & 512.30788 & 256.65758 & $\mathrm{~L}$ & 696.30551 & 348.65639 & 5 \\
6 & 691.31534 & 346.16131 & C-DeStreak & 583.22144 & 292.11436 & 4 \\
7 & 820.35794 & 410.68261 & $\mathrm{E}$ & 404.21397 & 202.61063 & 3 \\
8 & 948.41651 & 474.71190 & $\mathrm{Q}$ & 275.17138 & 138.08933 & 2 \\
9 & & & $\mathrm{~K}$ & 147.11280 & 74.06004 & 1 \\
\hline
\end{tabular}

(a)

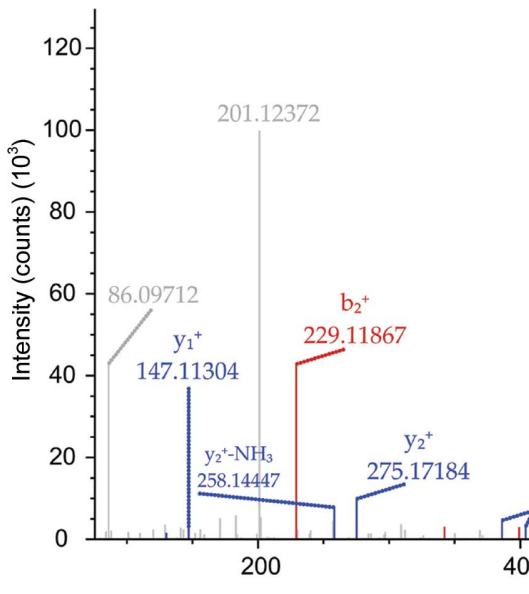

Parent $\mathrm{m} / \mathrm{z}=547.76489, \mathrm{RT}=49.1818 \mathrm{~min}, \mathrm{z}=+2$ DLLGLC(+75.99)EQK Cys528+BME

Figure 2

Determination of the Cys528 DeStreak modification of human CRM1 with 2-mercaptoethanol (BME) using mass-spectrometric analysis. (a) List of possible fragment ions of the Cys528-BME-containing peptide [DLLGLC $(+75.99) E Q K]$ with detected y ions in blue and b ions in red. $(b)$ Representative fragmentation spectrum (1 of 15) of the respective precursor ion with an $\mathrm{m} / \mathrm{z}$ of 547.765 . RT, retention time. 


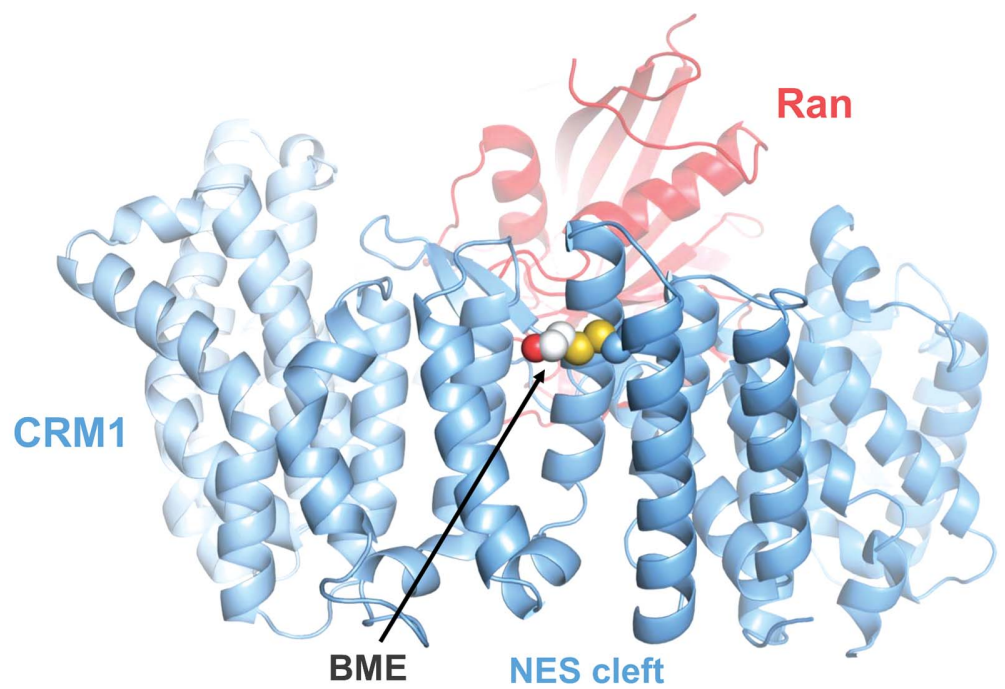

(a)

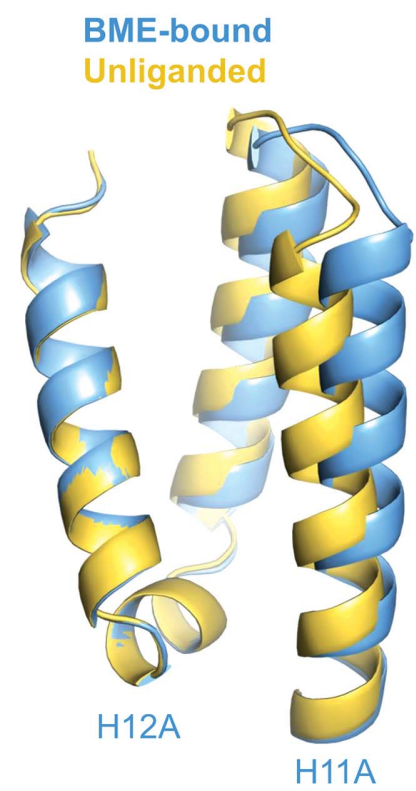

(b)
BME-bound

LMB-bound

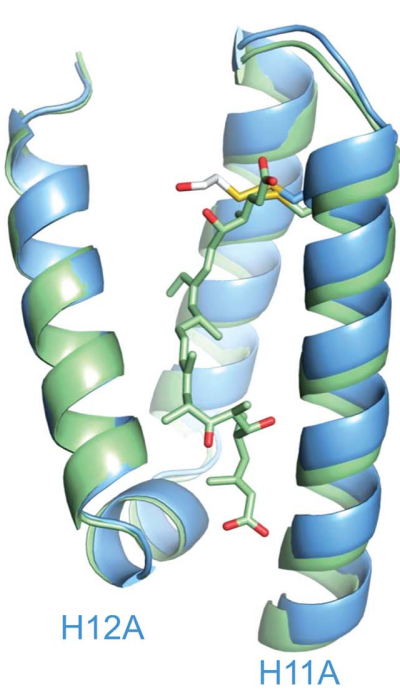

(c)
BME-bound
NES-bound

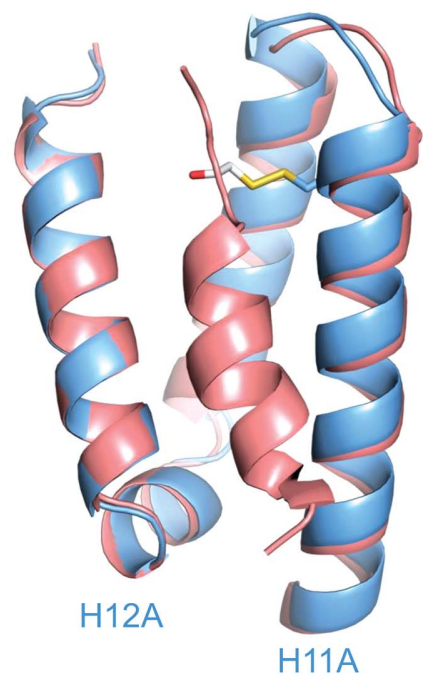

(d)

Figure 3

(a) Overall structure of the ${ }^{H s} \mathrm{CRM} 1-{ }^{H s}$ RanGTP-BME complex depicted in cartoon representation. CRM1 is shown in light blue and Ran is colored red. The BME-Cys528 covalent adduct, located in the NES-binding cleft, is represented as spheres. Structural alignment of the BME-bound cleft with the unliganded cleft (yellow; PDB entry 3m1i; Koyama \& Matsuura, 2010) is shown in (b), with LMB-bound CRM1 (pale green; PDB entry 6tvo; Shaikhqasem et al., 2020) in (c) and with NES-bound CRM1 (salmon; PDB entry 3nby; Güttler et al., 2010) in (d). BME is not shown in (b) and is depicted as sticks in $(c)$ and in $(d)$. LMB is depicted as sticks (pale green) in $(c)$.

inhibitor prior to crystallization seems to be sufficient for LMB to irreversibly react with the cysteine, as it binds in the nanomolar range $\left(\mathrm{IC}_{50}=151 \mathrm{n} M\right.$; Shaikhqasem et al., 2020). The resulting stable complex prohibits the possible modification by BME upon subsequent mixing with the crystallization buffer. In contrast, C6 demonstrates weaker binding than LMB $\left(\mathrm{IC}_{50}=4.2 \mu M\right.$; Shaikhqasem et al., 2020), and due to the possibility of its reversible binding such persistent stability could not be obtained.

Taken together, the presence of BME hinders the binding of the ligand of interest and thereby interferes with the crystallization of CRM1 in complex with novel inhibitor compounds. This interference can be avoided by elimination of BME from the protein-preparation buffer or by screening for a different crystallization condition with a lower $\mathrm{pH}$. Alternatively, undesired modification might be avoided by using reducing agents that demonstrate a higher stability than BME over a wider $\mathrm{pH}$ range such as, for example, tris(2-carboxyethyl)phosphine (TCEP; Getz et al., 1999).

\section{Conclusion}

Here, we have presented the crystal structure of human CRM1 modified at Cys528 by BME, which was used as a buffer component during protein purification. The detected modification hindered the binding of the inhibitor compound, which 
could not be localized in the electron-density map. In conclusion, our study provides another example of how protein buffer components and conditions can significantly interfere with the characterization of cysteine modifications. Therefore, buffer composition and chemical conditions must be critically considered during protein preparation and further experimentation.

\section{Acknowledgements}

The synchrotron X-ray diffraction data were collected on beamline P13 operated by EMBL Hamburg at the PETRA III storage ring, DESY, Hamburg, Germany. We would like to thank Dr David von Stetten for assistance in using the beamline. We are also grateful to Dr Piotr Neumann for critical reading of the manuscript and helpful discussions. Open access funding enabled and organized by Projekt DEAL.

\section{Funding information}

This work was supported by the DFG (SFB 860 TPB08 and INST186/1117).

\section{References}

Bhatnagar, A. \& Bandyopadhyay, D. (2018). Proteins, 86, 192-209.

Bonazzi, S., Eidam, O., Güttinger, S., Wach, J.-Y., Zemp, I., Kutay, U. \& Gademann, K. (2010). J. Am. Chem. Soc. 132, 1432-1442.

Cour, T. la, Kiemer, L., Mølgaard, A., Gupta, R., Skriver, K. \& Brunak, S. (2004). Protein Eng. Des. Sel. 17, 527-536.

Cox, J. \& Mann, M. (2008). Nat. Biotechnol. 26, 1367-1372.

Daelemans, D., Afonina, E., Nilsson, J., Werner, G., Kjems, J., De Clercq, E., Pavlakis, G. N. \& Vandamme, A.-M. (2002). Proc. Natl Acad. Sci. USA, 99, 14440-14445.

Dickmanns, A., Monecke, T. \& Ficner, R. (2015). Cells, 4, 538-568.

Dong, X., Biswas, A., Süel, K. E., Jackson, L. K., Martinez, R., Gu, H. \& Chook, Y. M. (2009). Nature, 458, 1136-1141.

Emsley, P., Lohkamp, B., Scott, W. G. \& Cowtan, K. (2010). Acta Cryst. D66, 486-501.

Etchin, J., Sun, Q., Kentsis, A., Farmer, A., Zhang, Z. C., Sanda, T., Mansour, M. R., Barcelo, C., McCauley, D., Kauffman, M., Shacham, S., Christie, A. L., Kung, A. L., Rodig, S. J., Chook, Y. M. \& Look, A. T. (2013). Leukemia, 27, 66-74.

Faustino, R. S., Nelson, T. J., Terzic, A. \& Perez-Terzic, C. (2007). Clin. Pharmacol. Ther. 81, 880-886.

Fetz, V., Knauer, S. K., Bier, C., von Kries, J. P. \& Stauber, R. H. (2009). Sensors, 9, 5423-5445.

Fung, H. Y. J., Fu, S.-C. \& Chook, Y. M. (2017). eLife, 6, e23961.

Getz, E. B., Xiao, M., Chakrabarty, T., Cooke, R. \& Selvin, P. R. (1999). Anal. Biochem. 273, 73-80.

Gorrec, F. (2009). J. Appl. Cryst. 42, 1035-1042.

Güttler, T. \& Görlich, D. (2011). EMBO J. 30, 3457-3474.

Güttler, T., Madl, T., Neumann, P., Deichsel, D., Corsini, L., Monecke, T., Ficner, R., Sattler, M. \& Görlich, D. (2010). Nat. Struct. Mol. Biol. 17, 1367-1376.

Haines, J. D., Herbin, O., de la Hera, B., Vidaurre, O. G., Moy, G. A., Sun, Q., Fung, H. Y. J., Albrecht, S., Alexandropoulos, K., McCauley, D., Chook, Y. M., Kuhlmann, T., Kidd, G. J., Shacham, S. \& Casaccia, P. (2015). Nat. Neurosci. 18, 511-520.

Hamamoto, T., Uozumi, T. \& Beppu, T. (1985). J. Antibiot. 38, 15731580.

Hill, R., Cautain, B., de Pedro, N. \& Link, W. (2014). Oncotarget, 5, $11-28$.
Hing, Z. A., Fung, H. Y. J., Ranganathan, P., Mitchell, S., El-Gamal, D., Woyach, J. A., Williams, K., Goettl, V. M., Smith, J., Yu, X., Meng, X., Sun, Q., Cagatay, T., Lehman, A. M., Lucas, D. M., Baloglu, E., Shacham, S., Kauffman, M. G., Byrd, J. C., Chook, Y. M., Garzon, R. \& Lapalombella, R. (2016). Leukemia, 30, 23642372.

Kabsch, W. (2010). Acta Cryst. D66, 133-144.

Kalid, O., Toledo Warshaviak, D., Shechter, S., Sherman, W. \& Shacham, S. (2012). J. Comput. Aided Mol. Des. 26, 1217-1228.

Kau, T. R., Schroeder, F., Ramaswamy, S., Wojciechowski, C. L., Zhao, J. J., Roberts, T. M., Clardy, J., Sellers, W. R. \& Silver, P. A. (2003). Cancer Cell, 4, 463-476.

Kim, H.-J., Ha, S., Lee, H. Y. \& Lee, K.-J. (2015). Mass Spectrom. Rev. 34, 184-208.

Kırlı, K., Karaca, S., Dehne, H. J., Samwer, M., Pan, K. T., Lenz, C., Urlaub, H. \& Görlich, D. (2015). eLife, 4, e11466.

Kleywegt, G. J. (1999). Acta Cryst. D55, 1878-1884.

Klomsiri, C., Karplus, P. A. \& Poole, L. B. (2011). Antioxid. Redox Signal. 14, 1065-1077.

Koyama, M. \& Matsuura, Y. (2010). EMBO J. 29, 2002-2013.

Kudo, N., Wolff, B., Sekimoto, T., Schreiner, E. P., Yoneda, Y., Yanagida, M., Horinouchi, S. \& Yoshida, M. (1998). Exp. Cell Res. 242, 540-547.

Lapalombella, R., Sun, Q., Williams, K., Tangeman, L., Jha, S., Zhong, Y., Goettl, V., Mahoney, E., Berglund, C., Gupta, S., Farmer, A., Mani, R., Johnson, A. J., Lucas, D., Mo, X., Daelemans, D., Sandanayaka, V., Shechter, S., McCauley, D., Shacham, S., Kauffman, M., Chook, Y. M. \& Byrd, J. C. (2012). Blood, 120, 4621-4634.

Liebschner, D., Afonine, P. V., Baker, M. L., Bunkóczi, G., Chen, V. B., Croll, T. I., Hintze, B., Hung, L.-W., Jain, S., McCoy, A. J., Moriarty, N. W., Oeffner, R. D., Poon, B. K., Prisant, M. G., Read, R. J., Richardson, J. S., Richardson, D. C., Sammito, M. D., Sobolev, O. V., Stockwell, D. H., Terwilliger, T. C., Urzhumtsev, A. G., Videau, L. L., Williams, C. J. \& Adams, P. D. (2019). Acta Cryst. D75, 861-877.

Liebschner, D., Afonine, P. V., Moriarty, N. W., Poon, B. K., Sobolev, O. V., Terwilliger, T. C. \& Adams, P. D. (2017). Acta Cryst. D73, 148-157.

Liu, X., Chong, Y., Liu, H., Han, Y. \& Niu, M. (2015). J. Ovarian Res. $\mathbf{8}, 35$.

Mathew, C. \& Ghildyal, R. (2017). Front. Microbiol. 8, 1171.

Mathur, S., Deshmukh, P., Tripathi, S., Marimuthu, P. \& Padmanabhan, B. (2018). J. Biomol. Struct. Dyn. 36, 2342-2360.

McCoy, A. J., Grosse-Kunstleve, R. W., Adams, P. D., Winn, M. D., Storoni, L. C. \& Read, R. J. (2007). J. Appl. Cryst. 40, 658-674.

Monecke, T., Güttler, T., Neumann, P., Dickmanns, A., Görlich, D. \& Ficner, R. (2009). Science, 324, 1087-1091.

Murshudov, G. N., Skubák, P., Lebedev, A. A., Pannu, N. S., Steiner, R. A., Nicholls, R. A., Winn, M. D., Long, F. \& Vagin, A. A. (2011). Acta Cryst. D67, 355-367.

Newlands, E. S., Rustin, G. J. \& Brampton, M. H. (1996). Br. J. Cancer, 74, 648-649.

Nicola, G., Fedarovich, A., Nicholas, R. A. \& Davies, C. (2005). Biochem. J. 392, 55-63.

Poole, L. B. (2015). Free Radical Biol. Med. 80, 148-157.

Port, S. A., Monecke, T., Dickmanns, A., Spillner, C., Hofele, R., Urlaub, H., Ficner, R. \& Kehlenbach, R. H. (2015). Cell Rep. 13, 690-702.

Rappsilber, J., Mann, M. \& Ishihama, Y. (2007). Nat. Protoc. 2, 1896 1906.

Sakakibara, K., Saito, N., Sato, T., Suzuki, A., Hasegawa, Y., Friedman, J. M., Kufe, D. W., VonHoff, D. D., Iwami, T. \& Kawabe, T. (2011). Blood, 118, 3922-3931.

Shaikhqasem, A., Dickmanns, A., Neumann, P. \& Ficner, R. (2020). J. Med. Chem. 63, 7545-7558.

Shevchenko, A., Wilm, M., Vorm, O. \& Mann, M. (1996). Anal. Chem. 68, 850-858. 
Sun, Q., Carrasco, Y. P., Hu, Y., Guo, X., Mirzaei, H., MacMillan, J. \& Chook, Y. M. (2013). Proc. Natl Acad. Sci. USA, 110, 13031308.

Tamura, S., Shimizu, N., Fujiwara, K., Kaneko, M., Kimura, T. \& Murakami, N. (2010). Bioorg. Med. Chem. Lett. 20, 2159-2162.

Tian, X., Gao, J., Liu, M., Lei, Y., Wang, F., Chen, J., Chu, P., Gao, J., Long, F., Liang, M., Long, X., Chu, H., Liu, C., Li, X., Sun, Q., Li, G. \& Yang, Y. (2020). J. Med. Chem. 63, 3881-3895.
Turner, J. G., Dawson, J. \& Sullivan, D. M. (2012). Biochem. Pharmacol. 83, 1021-1032.

Tyanova, S., Temu, T., Sinitcyn, P., Carlson, A., Hein, M. Y., Geiger, T., Mann, M. \& Cox, J. (2016). Nat. Methods, 13, 731-740.

Watt, P. J. van der \& Leaner, V. D. (2010). Clin. Cancer Res. 16, A33. Wingfield, P. T. (1995). Curr. Protoc. Protein Sci. 00, A.3A.1-A.3A.4. Zhukov, I., Ejchart, A. \& Bierzyński, A. (2008). Biochemistry, 47, 640-650. 